mining area in the Philippines which is the area of Itogon, Benguet.

Methods There were 93 small-scale miners who were included in the study as they complied with the inclusion criteria. The methods consisted of survey questionnaires, health physical examination guide, individual interviews, and work process observation tool.

Results The results showed that the small-scale miners worked for an average of 10.7 years, and a maximum work year of 40. The most widely employed mining technique was the dog-hole mining consisting of several sub-processes -tunneling, ball milling and gravity concentration, cyanide leaching, and smelting. The ergonomic and safety hazards identified were noise exposure from the dynamite blast, temperature extremes, and exposure to dust from dynamite blasting. The miners experienced prolonged crouching and bending, prolonged handling of tools, and carrying heavy sacks filled with mineral ores. In the ball milling and gravity concentration process, machine-related accidents were noted such as experiencing cuts from the crusher. In the cyanide leaching which uses massive amounts of cyanide, the most prevalent hazards were heat, dust, and chemicals such as cyanide fumes. In the smelting process, smoke from burning ore and coal as well as exposure to borax and nitric acid fumes. Burn injuries were reported among miners. A third (31.2\%) of miners have experienced accidents. The most common injury was laceration at $47.8 \%$, followed by methane inhalation, fracture of hand digits, and contusion at $17.4 \%$.

Conclusion The most prevalent health symptom reported by the miners was muscle pain which points to exposure to ergonomic hazards and risks.

\section{0-120 UNDER-REPORTING OF NON-FATAL OCCUPATIONAL INJURIES AMONG PRECARIOUS AND NON-PRECARIOUS WORKERS IN SWEDEN}

'Bertina Kreshpaj, Theo Bodin, David H Wegman, Nuria Matilla-Santander, Bo Burström, Gun Johansson, Katarina Kjellberg, Letitia Davis, Tomas Hemmingsson, Johanna Jonsson, Carin Håkansta, Cecilia Orellana. ${ }^{1}$ Karolinska Institutet, Sweden

\subsection{6/OEM-2021-EPI.63}

Introduction Data are lacking on under-reporting of occupational injuries (OI) among precariously employed workers in Sweden, challenging effective surveillance of OI and targeted preventive measures.

Objective To estimate the magnitude of under-reporting of OI among precarious and non-precarious workers in Sweden in 2013.

Methods Capture-recapture methods were applied using the national OI register and records from a labour market insurance company. All employed workers 18-65 resident in Sweden in 2013 were included in the study. Injuries were linked using personal identification numbers. Employment data were obtained from the national labour market register to construct precarious employment level, while injury severity (no healthcare/only outpatient/hospitalised) was constructed with data from the National Patient Register. Under-reporting estimates were computed stratifying by OI severity and by sociodemographic factors, occupation and precarious employment level.

Results Overall, under-reporting of OI was consistently higher across all socio-demographic factors for the very precarious group (very PER), followed by the precarious group (PER) and lastly the standard employment relationship group (SER). Under-reporting was higher among females compared to males (17.8\%, 95\% confidence interval (CI) 17.4-18.3), and younger compared to older workers $(19.8 \%, 95 \%$ CI 18.6-21). Notably under-reporting increased with educational level across all employment groups (20.9\%, 95\% CI 20.0-21.9). Underreporting of the OI decreased as injury severity increased and was higher with highest level of precariousness in all groups of severity.

Conclusions This is the first register-based study in Sweden to empirically demonstrate that under-reporting of $\mathrm{OI}$ is higher among precariously employed workers. OI under-reporting may represent unrecognized injuries that especially burden precariously employed workers' financial, health and social outcomes, shifting consequences from the employer to the employee.

\section{0-157 EFFECT OF EXPOSURE TO PSYCHOSOCIAL WORK FACTORS ON THE OCCURRENCE OF WORKPLACE INJURIES}

${ }^{1}$ Stephanie Boini, Regis Colin, Pascal Wild, Christophe Paris. 'Institut national de recherche et de sécurité (INRS), France

\subsection{6/OEM-2021-EPI.64}

Objective To determine the effect of psychosocial factors (PSF) on the incidence of workplace injuries (WI) among workers from of the French two-waves survey 'Health and Career paths'. A second objective was to explore effects of gender and age.

Methodology The outcome considered here was the occurrence of WI between 2006 and 2010, reported by participants. Nineteen self-reported PSF, evaluated in 2006, explored six domains: labour intensity and working time (9 items), emotional demand (3), autonomy (2), social relationships at work (2), conflict of values (2), and job insecurity (1). Multiple logistic models were performed for each PSF. Interactions between PSF and respectively gender and age class $(\leq 30$ y., 30 $50 \mathrm{y}$., $\geq 50 \mathrm{y}$.) were tested using Wald test.

Results The study population consists in 3,277 men and 3,565 women in employment in 2006 and re-interviewed in 2010. Over the period, 255 workers reported at least one WI, corresponding to an annual average rate of $6 \mathrm{WI} / 1000$ workers. PSF associated with the incidence of WI were mostly related to emotional demand, social relationship at work and labour intensity. The risk of WI was greater in women reporting difficulties reconciling work and family life, tensions with public, necessity to hide or fake emotions or lack of reward, and in men reporting more than $48 \mathrm{~h} /$ week, irregular working hours or fear for own safety or for safety for others during work. Participants under 30y. reporting tensions with public, necessity to hide or fake emotions, or fear for own safety or for safety for others during work had a greater risk of WI than the others.

Conclusion These results provide evidence for relationships between PSF exposure and the occurrence of WI. In particular, the risk of WI was greater in participants reporting exposure to emotional demand at work and varied according to gender and age. 